\title{
Traveling Wavefronts of a Diffusive Hematopoiesis Model with Time Delay
}

\author{
Zhi Ling*, Linling Zhu \\ School of Mathematical Science, Yangzhou University, Yangzhou, China \\ Email: zhling@yzu.edu.cn, 819351352@qq.com
}

Received 16 July 2014; revised 8 August 2014; accepted 1 September 2014

Copyright (C) 2014 by authors and Scientific Research Publishing Inc.

This work is licensed under the Creative Commons Attribution International License (CC BY). http://creativecommons.org/licenses/by/4.0/

c) (i) Open Access

\begin{abstract}
In this paper, a reaction-diffusion equation with discrete time delay that describes the dynamics of the blood cell production is analyzed. The existence of the traveling wave front solutions is demonstrated using the technique of upper and lower solutions and the associated monotone iteration.
\end{abstract}

\section{Keywords}

Traveling Wavefronts, Hematopoiesis Model, Time Delay

\section{Introduction}

It is well know that the traveling wave theory was initiated in 1937 by Kolmogorov, Petrovskii, Piskunov [1] and Fisher [2]. Now, the theory of traveling wave solutions to reaction-diffusion equations is one of the fast developing areas of modern mathematics and has attracted much attention due to its significance in biology, chemistry, epidemiology and physics, see [3] [4] and the reference cited therein. In recent years, the traveling wave problem for reaction-diffusion systems with delay has been widely studied. For example, Gomez and Trofimchuk [5] considered the Fisher-KPP equation and their results showed that each monotone traveling wave could be found via an iteration procedure by using the special montone integral operators. Schaaf [6] systematically studied two scalar reaction-diffusion equations with a single discrete delay by using the phase plane technique, the maximum principle for parabolic functional differential equations and the general theory of ordinary differential equations. The degree theory has been adopted in [7] [8].

In this paper we consider the following reaction-diffusion equation with a discrete time delay:

$$
\frac{\partial N(t, x)}{\partial t}=\Delta N(t, x)-\delta N(t, x)+\frac{\beta N(t-\tau, x)}{1+N^{n}(t-\tau, x)} .
$$

${ }^{*}$ Corresponding author. 
When $N$ is independent on the spatial variable $x$, the above equation reduces to the following ordinary differential equation

$$
N^{\prime}(t)=-\delta N(t)+\frac{\beta N(t-\tau)}{1+N^{n}(t-\tau)},
$$

which was first proposed by Mackey and Glass [9] to describe the dynamics of blood cell production. Here, $N(t)$ denotes the density of mature stem cells in blood circulation and $\tau$ is the time delay between the production of immature stem cells in bone marrow and their maturation for release in the circulating blood stream; $\delta, \beta \in(0,+\infty)$ and $n \in(1,+\infty)$ are positive constants that represent some specific meanings in blood circulation. For instance, $\delta$ is the lost rate of the cells from the circulation. The term $\frac{\beta N(t-\tau)}{1+N^{n}(t-\tau)}$ shows that the flux of the cells into the circulation from the stem cell compartment depends on the number of cells $N$ at time $t-\tau$. For more details about Hematopoiesis model, we refer the readers to the articles of Mackey [9]-[11] and the references given in them.

Equation (1.2) has been studied by many authors such as in [12]-[14]. Weng and Dai [13] proved that the positive equilibrium to Equation (1.2) could be a global attractor under some conditions. Wu, Li and Zhou [14] derived a sufficient and necessary condition that guarantees the existence of positive periodic solutions of Equation (1.2) with periodic coefficients.

Equation (1.2) can be generalized as the following functional differential equation

$$
\frac{\partial u(t, x)}{\partial t}-\Delta u(t, x)=-\delta u(t, x)+f(u(t-\tau, x)) .
$$

Wang [15] investigated the generalized equation with Neumann boundary condition and obtained the oscillatory behavior of solutions about the positive equilibrium of (1.3). Further, they derived the sufficient and necessary conditions for global attractivity of the zero solution. In addition, global attractivity of the positive equilibrium of (1.3) was investigated by Gopalsamy and Kulenvic [16]. Cheng and Zhang [17] and Jiang et al. [18] ( $n$-dimentional case) instead investigated the existence of positive periodic solutions of Equation (1.3) by using the Krasnosel skii fixed point theorem.

The aim of this paper is to consider the existence of traveling wave front solutions for (1.1) in the case of one dimensional space.

This paper is outlined as follows. The next section, we will introduce the technique of upper and lower solutions developed by Wu and Zou [19]. The conditions for establishing the positive equilibria and obtaining the existence of traveling waves are derived in Section 2.

To investigate the existence of traveling wave fronts of (1.1), we describe briefly the technique of upper and lower solutions developed by $\mathrm{Wu}$ and Zhou [19].

Consider a scalar reaction-diffusion equation with time delay:

$$
\frac{\partial u(t, x)}{\partial t}=D \frac{\partial u^{2}(t, x)}{\partial x^{2}}+f\left(u_{t}(x)\right)
$$

where $t \geq 0, x \in \mathbb{R}, u \in \mathbb{R}$, and $D>0$ is the diffusion coefficient. The function $f: C([-\tau, 0], \mathbb{R}) \rightarrow \mathbb{R}$ is continuous and $u_{t}(x)$ is an element in $C([-\tau, 0], \mathbb{R}) \rightarrow \mathbb{R}$ parameterized by $x \in \mathbb{R}$ and given by

$$
u_{t}(x)(s)=u(t+s, x), \quad s \in[-\tau, 0], t \geq 0, x \in \mathbb{R} .
$$

Looking for traveling wave solutions of the form $u(t, x)=\phi(x+c t)$ leads to a second-order functional differential Equation

$$
D \phi^{\prime \prime}(t)-c \phi^{\prime}(t)+f_{c}\left(\phi_{t}\right)=0, \quad t \in \mathbb{R},
$$

where $f_{c}: X_{c} \triangleq C([-c \tau, 0], \mathbb{R}) \rightarrow \mathbb{R}$ is define by

$$
f_{c}(\psi)=f\left(\psi^{c}\right), \quad \psi^{c}(s)=\psi(c s), \quad s \in[-\tau, 0] .
$$


Now we assume that

A1. There exists $K>0$ such that $f_{c}(\hat{0})=f_{c}(\hat{K})=0$ and $f_{c}(\hat{u}) \neq 0$ for $u \in(0, K)$, where $\hat{u}$ denotes the constant function taking the value $u$ on $[-c \tau, 0]$.

A2. There exists $\alpha \geq 0$ such that

$$
f_{c}(\phi)-f_{c}(\psi)+\alpha[\phi(0)-\psi(0)] \geq 0
$$

for $\phi, \psi \in X_{c}$ with $0 \leq \psi(s) \leq \phi(s) \leq K, s \in[-c \tau, 0]$.

If for some $c>0,(1.5)$ has a monotone solution $\phi$ defined on $\mathbb{R}$ such that

$$
\lim _{t \rightarrow-\infty} \phi(t)=0, \quad \lim _{t \rightarrow \infty} \phi(t)=K,
$$

then $u(t, x)=\phi(x+c t)$ is called a traveling wave front of (1.4) with a wave speed $c$.

Define a profile set for traveling wave fronts of (1.1) by

$$
\Gamma=\left\{\phi \in C\left(\mathbb{R}, \mathbb{R}^{n}\right): \begin{array}{l}
(i) \phi \text { is nondecreasing in } \mathbb{R}, \\
\text { (ii) } \lim _{t \rightarrow-\infty} \phi(t)=0, \lim _{t \rightarrow \infty} \phi(t)=K,
\end{array}\right\} .
$$

The upper and lower solution for (2.1) are defined as follows:

Definition 1 The piecewise smooth functions $\bar{\phi}$ and $\phi$ in $C(\mathbb{R}, \mathbb{R})$ are called upper and lower solution of (1.4) if $\bar{\phi} \geq \underline{\phi}$ and if

$$
c \bar{\phi}^{\prime}(t) \geq D \bar{\phi}^{\prime \prime}(t)+f_{c}\left(\bar{\phi}_{t}\right), \quad t \in \mathbb{R}
$$

and $\phi$ satisfies the above differential inequalities in reversed order.

Now we are in the position to state a scalar version of [19] (Theorem 3.6).

Theorem 1 If the conditions (A1) and (A2) hold, suppose that (1.5) has an upper solution $\bar{\phi}$ in $\Gamma$ and a lower solution $\phi$ (which is not necessarily in $\Gamma$ ) with $0 \leq \phi(t) \leq \bar{\phi}(t) \leq K$ and $\phi(t) \neq \equiv$ in $\mathbb{R}$, then the problem (1.4) admits a traveling wave front.

\section{Existence of Traveling Wave Fronts}

Assume that $\beta>\delta$, and we can get two equilibria of (1.1)

$$
k_{1}=0, \quad k_{2}=\left(\frac{\beta}{\delta}-1\right)^{\frac{1}{n}}>0 .
$$

We will tackle the existence of solutions of (3.1) with the asymptotic boundary condition

$$
\lim _{t \rightarrow-\infty} \phi(t)=k_{1}, \quad \lim _{t \rightarrow-\infty} \phi(t)=k_{2},
$$

which corresponds to the traveling wave fronts of (1.2) connecting $k_{1}$ and $k_{2}$.

Substituting $u(t, x)=\phi(s)$ into (1.2), and denoting the movingvariable $s$ still by $t$, the resulting wave equation becomes

$$
c \phi^{\prime}(t)-\phi^{\prime \prime}(t)=-\delta \phi(t)+\frac{\beta \phi(t-c \tau)}{1+\phi^{n}(t-c \tau)} .
$$

Define the function

$$
f_{c}(\phi)=-\delta \phi(0)+\frac{\beta \phi(c \tau)}{1+\phi^{n}(c \tau)} .
$$

Lemma 1 If $\beta>\delta$, then $f_{c}(\hat{0})=f_{c}\left(\hat{k}_{2}\right)=0$, and $f_{c}(\hat{K}) \neq 0$, for any $K \in\left(k_{1}, k_{2}\right)$, where $\hat{K}$ denotes the constant function taking the value $K$ on $[-c \tau, 0]$.

Next we show that $f_{c}(\phi)$ satisfies quasi-monotonicity condition with some assumptions. 
Lemma 2 If $\frac{n}{n-1}>\frac{\beta}{\delta}>1, n \in(1, \infty)$, then $f_{c}(\phi)$ satisfies the following quasi-monotonicity condition:

Take $\alpha \geq \delta$, and we have

$$
f_{c}(\phi)-f_{c}(\psi)+\alpha[\phi(0)-\psi(0)] \geq 0
$$

for all $\phi, \psi \in C([-c \tau, 0], \mathbb{R})$ with $k_{1} \leq \psi(s) \leq \phi(s) \leq k_{2}, \quad s \in[-c \tau, 0]$.

Proof 1 Consider the function $h(y)=\frac{y}{1+y^{n}}$, and it is obvious that

$$
h^{\prime}(y)=\frac{1+(1-n) y^{n}}{\left(1+y^{n}\right)^{2}} \geq 0, \text { for } 0 \leq y \leq\left(\frac{1}{n-1}\right)^{\frac{1}{n}} .
$$

If $\frac{n}{n-1}>\frac{\beta}{\delta}>1$, then

$$
0 \leq \psi(t) \leq \phi(t) \leq k_{2}=\left(\frac{\beta}{\delta}-1\right)^{\frac{1}{n}} \leq\left(\frac{1}{n-1}\right)^{\frac{1}{n}}
$$

since $n>1$.

It demonstrates that the function $h(y)$ is increasing on $\left[k_{1}, k_{2}\right]$. A direct computation shows that

$$
\begin{aligned}
f_{c}(\phi)-f_{c}(\psi) & =-\delta \phi(0)+\frac{\beta \phi(c \tau)}{1+\phi^{n}(c \tau)}+\delta \psi(0)-\frac{\beta \psi(c \tau)}{1+\psi^{n}(c \tau)} \\
& =-\delta(\phi(0)-\psi(0))+\beta\left(\frac{\phi(c \tau)}{1+\phi^{n}(c \tau)}-\frac{\psi(c \tau)}{1+\psi^{n}(c \tau)}\right) \\
& \geq-\delta(\phi(0)-\psi(0)),
\end{aligned}
$$

and then

$$
f_{c}(\phi)-f_{c}(\psi)+\alpha(\phi(0)-\psi(0)) \geq(\alpha-\delta)(\phi(0)-\psi(0)) .
$$

Therefore, if choosing $\alpha \geq \delta$, we have

$$
f_{c}(\phi)-f_{c}(\psi)+\alpha(\phi(0)-\psi(0)) \geq 0 .
$$

This completes the proof.

Remark 1 If $n=1$, the function $h(y)=\frac{y}{1+y}$ is increasing for all $y>0$. When $\beta>\delta$ the condition (A2) is hold.

Define the profile set

$$
\Gamma^{*}=\left\{\phi \in C(\mathbb{R}, \mathbb{R}): \begin{array}{l}
\text { (i) } \phi \text { is nondecreasing in } \mathbb{R}, \\
(\text { ii }) \lim _{t \rightarrow-\infty} \phi(t)=k_{1}, \lim _{t \rightarrow \infty} \phi(t)=k_{2},
\end{array}\right\} .
$$

Next we will discuss the existence problem by using the method of upper and lower solutions that are defined as follows:

Definition 2 The piecewise smooth functions $\bar{\phi}$ and $\phi$ in $C(\mathbb{R}, \mathbb{R})$ are called upper and lower solution of (3.1) if $\bar{\phi} \geq \underline{\phi}$ and if 


$$
c \bar{\phi}^{\prime}(t)-\bar{\phi}^{\prime \prime}(t) \geq-\delta \bar{\phi}(t)+\frac{\beta \bar{\phi}(t-c \tau)}{1+\bar{\phi}^{n}(t-c \tau)} \text { in } \mathbb{R}
$$

and $\phi$ satisfies the above differential inequalities in reversed order.

Define

$$
\Delta_{c}(\lambda)=\lambda^{2}-c \lambda-\delta+\beta \mathrm{e}^{-c \lambda \tau},
$$

then we have the following lemmas.

Lemma 3 There exists $c^{*}>0$ such that for $c>c^{*}, \Delta_{c}(\lambda)=0$ has two positive real roots, $0<\lambda_{1}<\lambda_{2}$ and

$$
\Delta_{c}(\lambda)= \begin{cases}>0, & \text { for } \lambda>\lambda_{2} \\ <0, & \text { for } \lambda \in\left(\lambda_{1}, \lambda_{2}\right), \\ >0, & \text { for } \lambda<\lambda_{1}\end{cases}
$$

Since the proof of this lemma is similar to that of Claim 2.3 of [19], we omit it. Next we first construct the upper solution of (3.1).

Lemma 4 Assume $c>c^{*}$, then $\bar{\phi}(t)=\min \left\{k_{2}, \mathrm{e}^{\lambda_{1} t}\right\}$ is an upper solution of (3.1) and $\bar{\phi} \in \Gamma^{*}$.

Proof 2 It is easy to verify that $\bar{\phi} \in \Gamma^{*}$. We show that $\bar{\phi}$ is an upper solution of (3.1).

Let $t_{0}$ be such that $\mathrm{e}^{\lambda_{1} t_{0}}=k_{2}$.

i) For $t \geq t_{0}, \bar{\phi}^{\prime}(t)=0, \bar{\phi}^{\prime \prime}(t)=0, \bar{\phi}(t)=k_{2}, \bar{\phi}(t-c \tau) \leq k_{2}$. thus

$$
\bar{\phi}^{\prime \prime}(t)-c \bar{\phi}^{\prime}(t)-\delta \bar{\phi}(t)+\frac{\beta \bar{\phi}(t-c \tau)}{1+\bar{\phi}^{n}(t-c \tau)} \leq \delta k_{2}+\frac{\beta \bar{\phi}(t-c \tau)}{1+\bar{\phi}^{n}(t-c \tau)} \leq 0 .
$$

ii) For $t<t_{0}, \bar{\phi}(t)=\mathrm{e}^{\lambda_{1} t}$ and $\bar{\phi}(t-c \tau)=\mathrm{e}^{\lambda_{1}(t-c \tau)}$, thus

$$
\begin{aligned}
\bar{\phi}^{\prime \prime}(t)-c \bar{\phi}^{\prime}(t)-\delta \bar{\phi}(t)+\frac{\beta \bar{\phi}(t-c \tau)}{1+\bar{\phi}^{n}(t-c \tau)} & \leq \overline{\phi^{\prime \prime}}(t)-c \overline{\phi^{\prime}}(t)-\delta \bar{\phi}(t)+\beta \bar{\phi}(t-c \tau) \\
& \leq \mathrm{e}^{\lambda_{1} t}\left(\lambda_{1}^{2}-c \lambda_{1}-\delta+\beta \mathrm{e}^{-c \lambda_{1} \tau}\right)=\mathrm{e}^{\lambda_{1} t} \Delta_{c}\left(\lambda_{1}\right)=0 .
\end{aligned}
$$

According to the discussion above, we know that $\bar{\phi}$ is an upper solution of (1.5). This completes the proof.

We now give the lower solution to (1.5). Let $c>c^{*}$ and $0<\lambda_{1}<\lambda_{2}$ be the same as those given in Lemma 3.2. Take $\varepsilon>0$ such that $\varepsilon<\lambda_{1}<\lambda_{1}+\varepsilon<\lambda_{2}$. Define $\underline{\phi}=\max \left\{0,\left(1-M \mathrm{e}^{\varepsilon t}\right) \mathrm{e}^{\lambda_{1} t}\right\}$, where the constant $M>1$ is to be determined.

Lemma 5 For $M>\frac{\beta \mathrm{e}^{-2 \lambda_{1} c \tau}\left(1+\mathrm{e}^{-\varepsilon c \tau}\right)^{2}}{-\Delta_{c}\left(\lambda_{1}+\varepsilon\right)}, \underline{\phi}(t)=\max \left\{0,\left(1-M \mathrm{e}^{\varepsilon t}\right) \mathrm{e}^{\lambda_{1} t}\right\}$ is a lower solution for Equation (3.1).

Proof 3 Let $t_{1}=\frac{1}{\varepsilon} \ln \left(\frac{1}{M}\right)$, then $t_{1}<0$ for $M>1$ and

$$
\underline{\phi}(t)= \begin{cases}0, & \text { for } t>t_{1}, \\ \left(1-M \mathrm{e}^{\varepsilon t}\right) \mathrm{e}^{\lambda_{1} t}, & \text { for } t<t_{1} .\end{cases}
$$

i) For $t>t_{1}, \underline{\phi}(t)=0, \underline{\phi}(t-c \tau)=0, \underline{\phi}^{\prime}(t)=0$, and $\underline{\phi}^{\prime \prime}(t)=0$. Hence

$$
c \underline{\phi}^{\prime}(t)-\underline{\phi}^{\prime \prime}(t)+\delta \underline{\phi}(t)-\frac{\beta \underline{\phi}(t-c \tau)}{1+\underline{\phi}^{n}(t-c \tau)}=0
$$

ii) For $t<t_{1}$, we have 


$$
\begin{aligned}
& \underline{\phi}^{\prime}(t)=\left[\lambda_{1}-M\left(\varepsilon+\lambda_{1}\right) \mathrm{e}^{\varepsilon t}\right] \mathrm{e}^{\lambda_{1} t}, \\
& \underline{\phi}^{\prime \prime}(t)=\left[\lambda_{1}^{2}-M\left(\varepsilon+\lambda_{1}\right)^{2} \mathrm{e}^{\varepsilon t}\right] \mathrm{e}^{\lambda_{1} t}, \\
& \underline{\phi}(t-c \tau)=\left[1-M \mathrm{e}^{\varepsilon(t-c \tau)}\right] \mathrm{e}^{\lambda_{1}(t-c \tau)} .
\end{aligned}
$$

It is easy to check that $0 \leq\left(1-M \mathrm{e}^{\varepsilon(t-c \tau)}\right) \mathrm{e}^{\lambda_{1}(t-c \tau)}<1$. It follows that $1+\underline{\phi}^{n}<\frac{1}{1-\underline{\phi}}$. Therefore

$$
\begin{aligned}
& \underline{\phi}^{\prime \prime}(t)-c \underline{\phi}^{\prime}(t)-\delta \underline{\phi}(t)+\frac{\beta \underline{\phi}(t-c \tau)}{1+\underline{\phi}^{n}(t-c \tau)} \\
& \geq \underline{\phi}^{\prime \prime}(t)-c \underline{\phi}^{\prime}(t)-\delta \underline{\phi}(t)+\beta \underline{\phi}(t-c \tau)\left[1-\underline{\phi}^{n}(t-c \tau)\right] \\
& =\left[\lambda_{1}^{2}-M\left(\varepsilon+\lambda_{1}\right)^{2} \mathrm{e}^{\varepsilon t}\right] \mathrm{e}^{\lambda_{1} t}-c\left[\lambda_{1}-M\left(\varepsilon+\lambda_{1}\right) \mathrm{e}^{\varepsilon t}\right] \mathrm{e}^{\lambda_{1} t}-\delta\left(1-M \mathrm{e}^{\varepsilon t}\right) \mathrm{e}^{\lambda_{1} t} \\
& +\beta\left[1-M \mathrm{e}^{\varepsilon(t-c \tau)}\right] \mathrm{e}^{\lambda_{1}(t-c \tau)}\left[1-\left(1-M \mathrm{e}^{\varepsilon(t-c \tau)}\right) \mathrm{e}^{\lambda_{1}(t-c \tau)}\right] \\
& =\mathrm{e}^{\lambda_{1} t}\left[\Delta_{c}\left(\lambda_{1}\right)-M \mathrm{e}^{\varepsilon} \Delta_{c}\left(\lambda_{1}+\varepsilon\right)-\beta \mathrm{e}^{-2 \lambda_{1} c \tau} \mathrm{e}^{\lambda_{1} \tau}\left(1-M \mathrm{e}^{\varepsilon(t-c \tau)}\right)\right] \\
& \geq \mathrm{e}^{\lambda_{1} t}\left[-M \mathrm{e}^{\varepsilon t} \Delta_{c}\left(\lambda_{1}+\varepsilon\right)-\beta \mathrm{e}^{-2 \lambda_{1} c \tau} \mathrm{e}^{\varepsilon t}\left(1-M \mathrm{e}^{\varepsilon(t-c \tau)}\right)\right] \\
& =\mathrm{e}^{\left(\lambda_{1}+\varepsilon\right) t}\left[-M \Delta_{c}\left(\lambda_{1}+\varepsilon\right)-\beta \mathrm{e}^{-2 \lambda_{1} c \tau}\left(1-M \mathrm{e}^{\varepsilon(t-c \tau)}\right)\right] .
\end{aligned}
$$

Note that

$$
\begin{aligned}
& 1-M \mathrm{e}^{\varepsilon(t-c \tau)}>1-M \mathrm{e}^{\varepsilon t_{1}} \mathrm{e}^{-\varepsilon c \tau}=1-\mathrm{e}^{-\varepsilon c \tau}, \\
& 1-M \mathrm{e}^{\varepsilon(t-c \tau)}<1+M \mathrm{e}^{\varepsilon t_{1}} \mathrm{e}^{-\varepsilon c \tau}<1+\mathrm{e}^{-\varepsilon c \tau} .
\end{aligned}
$$

Since $t<t_{1}<0, \varepsilon<\lambda_{1}$, we have $\left[1-M \mathrm{e}^{\varepsilon(t-c \tau)}\right]^{2}<\left(1-\mathrm{e}^{\varepsilon c \tau}\right)^{2}$. Thus

$$
\begin{aligned}
\underline{\phi^{\prime \prime}}(t)-c \underline{\phi^{\prime}}(t)-\delta \underline{\phi}(t)+\frac{\beta \underline{\phi}(t-c \tau)}{1+\underline{\phi}^{n}(t-c \tau)} & =\mathrm{e}^{\left(\lambda_{1}+\varepsilon\right) t}\left[-M \Delta_{c}\left(\lambda_{1}+\varepsilon\right)-\beta \mathrm{e}^{-2 \lambda_{1} c \tau}\left(1-M \mathrm{e}^{\varepsilon(t-c \tau)}\right)\right] \\
& \geq \mathrm{e}^{\left(\lambda_{1}+\varepsilon\right) t}\left[-M \Delta_{c}\left(\lambda_{1}+\varepsilon\right)\right]\left[M-\frac{\beta \mathrm{e}^{-2 \lambda_{1} c \tau}\left(1+\mathrm{e}^{-\varepsilon c \tau}\right)^{2}}{-\Delta_{c}\left(\lambda_{1}+\varepsilon\right)}\right] .
\end{aligned}
$$

If we choose

$$
M>\frac{\beta \mathrm{e}^{-2 \lambda_{1} c \tau}\left(1+\mathrm{e}^{-\varepsilon c \tau}\right)^{2}}{-\Delta_{c}\left(\lambda_{1}+\varepsilon\right)},
$$

then $\underline{\phi}^{\prime \prime}(t)-c \underline{\phi^{\prime}}(t)-\delta \underline{\phi}(t)+\frac{\beta \underline{\phi}(t-c \tau)}{1+\underline{\phi}^{n}(t-c \tau)} \geq 0$. Thus $\underline{\phi}$ is a lower solution of (3.1).

It is clear that $k_{1} \leq \underline{\phi} \leq \bar{\phi} \leq k_{2}$. Summarizing the above conclusions, we give our main result of this paper below.

Theorem 2 If $\frac{n}{n-1}>\frac{\beta}{\delta}>1, n \in(1, \infty)$, or $n=1, \beta>\delta$, then for every $c>c^{*}$, the problem (1.2) has a traveling wave front which connects the equilibria $k_{1}=0$ and $k_{2}=\left[\frac{\beta}{\delta}-1\right]^{\frac{1}{n}}$. 


\section{References}

[1] Kolmogorov, A., Petrovskii, I. and Piskunov, N. (1937) Study of a Diffusion Equation That Is Related to the Growth of a Quality of Matter and Its Application to a Biological Problem. Moscow University Mathematics Bulletin, 1, 1-26.

[2] Fisher, R.A. (1937) The Wave of Advance of Advantageous Gene. Annals of Eugenics, 7, 355-369. http://dx.doi.org/10.1111/j.1469-1809.1937.tb02153.x

[3] Murray, J.D. (1989) Mathematical Biology. Springer, New York. http://dx.doi.org/10.1007/978-3-662-08539-4

[4] Volpert, A.I. and Volpert, V.A. (1994) Traveling Wave Solutions of Parabolic Systems, Translation of Mathematical Monographs. American Mathematical Society, RI, 140.

[5] Gomez, A. and Trofimchuk, S. (2011) Monotone Traveling Wavefronts of the KPP-Fisher Delayed Equation. Journal of Differential Equations, 250, 1767-1787. http://dx.doi.org/10.1016/j.jde.2010.11.011

[6] Schaaf, K. (1987) Asymptotic Behavior and Traveling Wave Solutions for Parabolic Functional Differential Equations. Transactions of the American Mathematical Society, 302, 587-615.

[7] Huang, J. and Zou, X. (2003) Existence of Travelling Wave Fronts of Delayed Reaction Diusion Systems without Monotonicity. Discrete and Continuous Dynamical Systems, 9, 925-936. http://dx.doi.org/10.3934/dcds.2003.9.925

[8] Ma, S. (2001) Travelling Wavefronts for Delayed Reaction-Diusion Systems via a Fixed Point Theorem. Journal of Differential Equations, 171, 294-314. http://dx.doi.org/10.1006/jdeq.2000.3846

[9] Mackey, M.C. and Glass, L. (1977) Oscillation and Chaos in Physiological Control System. Science, 197, $287-289$. http://dx.doi.org/10.1126/science.267326

[10] Mackey, M.C. (1978) Unified Hypothesis for the Origin of Aplastic Anemia and Periodic Hematopoiesis. Blood, 51, 941-956.

[11] Mackey, M.C. (1981) Some Models in Hemapoiesis: Predictions and Problems. In: Biomathematics and Cell Kinetzcs, Elservier, North Holland, 23-38.

[12] Liz, E., Tkachenko, V. and Trofimchuk, S. (2003) A Global Stability Criterion for Scalar Functional Differential Equations. SIAM Journal on Mathematical Analysis, 35, 596-622. http://dx.doi.org/10.1137/S0036141001399222

[13] Weng, P.X. and Dai, Z.P. (2001) Global Atractivity for a Model of Hemapoiesis. Journal of South China Normal University, 2, 12-19.

[14] Wu, X.M., Li, J.W. and Zhou, H.Q. (2007) A Necessary and Sufficient Condition for the Existence of Positive Periodic Solutions of a Model of Hematopoiesis. Computers \& Mathematics with Applications, 54, 840-849. http://dx.doi.org/10.1016/j.camwa.2007.03.004

[15] Wang, X. and Li, Z.X. (2007) Dynamics for a Type of General Reaction-Diffusion Model. Nonlinear Analysis: Theory, Methods \& Applications, 67, 2699-2711. http://dx.doi.org/10.1016/j.na.2006.09.034

[16] Gopalsamy, K., Kulenvic, M.R. and Ladas, G. (1990) Oscillations and Global Attractivity in Models of Hematopoiesis. Journal of Dynamics and Differential Equations, 2, 117-132. http://dx.doi.org/10.1007/BF01057415

[17] Cheng, S.S. and Zhang, G. (2001) Existence of Positive Periodic Solutions for Non-Autonomaous Functional Differential Equations. Electronic Journal of Differential Equations, 59, 1-8.

[18] Jiang, D.Q., Wei, J.J. and Zhang, B. (2002) Positive Periodic Solutions of Functional Differential Equations and Population Model. Electronic Journal of Differential Equations, 71, 1-13.

[19] Wu, J.H. and Zou, X.F. (2001) Traveling Wave Fronts of Reaction-Diffusion Systems. Journal of Dynamics and Differential Equations, 13, 651-687. http://dx.doi.org/10.1023/A:1016690424892 
Scientific Research Publishing (SCIRP) is one of the largest Open Access journal publishers. It is currently publishing more than 200 open access, online, peer-reviewed journals covering a wide range of academic disciplines. SCIRP serves the worldwide academic communities and contributes to the progress and application of science with its publication.

Other selected journals from SCIRP are listed as below. Submit your manuscript to us via either submit@scirp.org or Online Submission Portal.
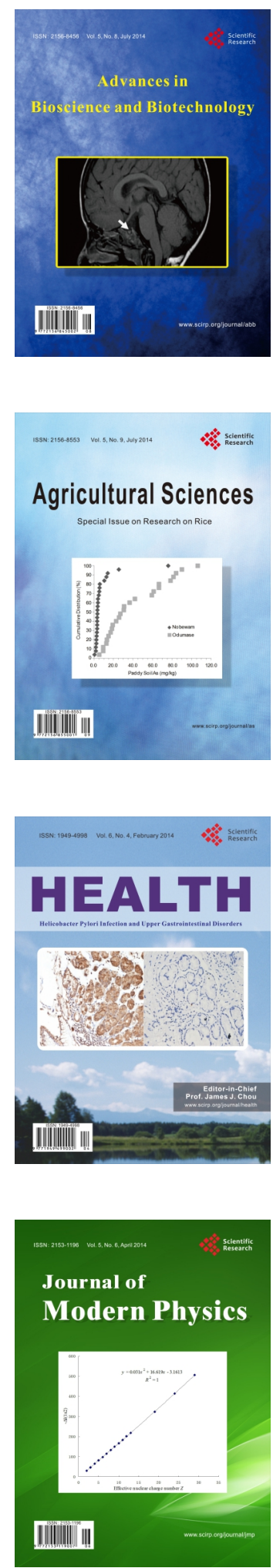
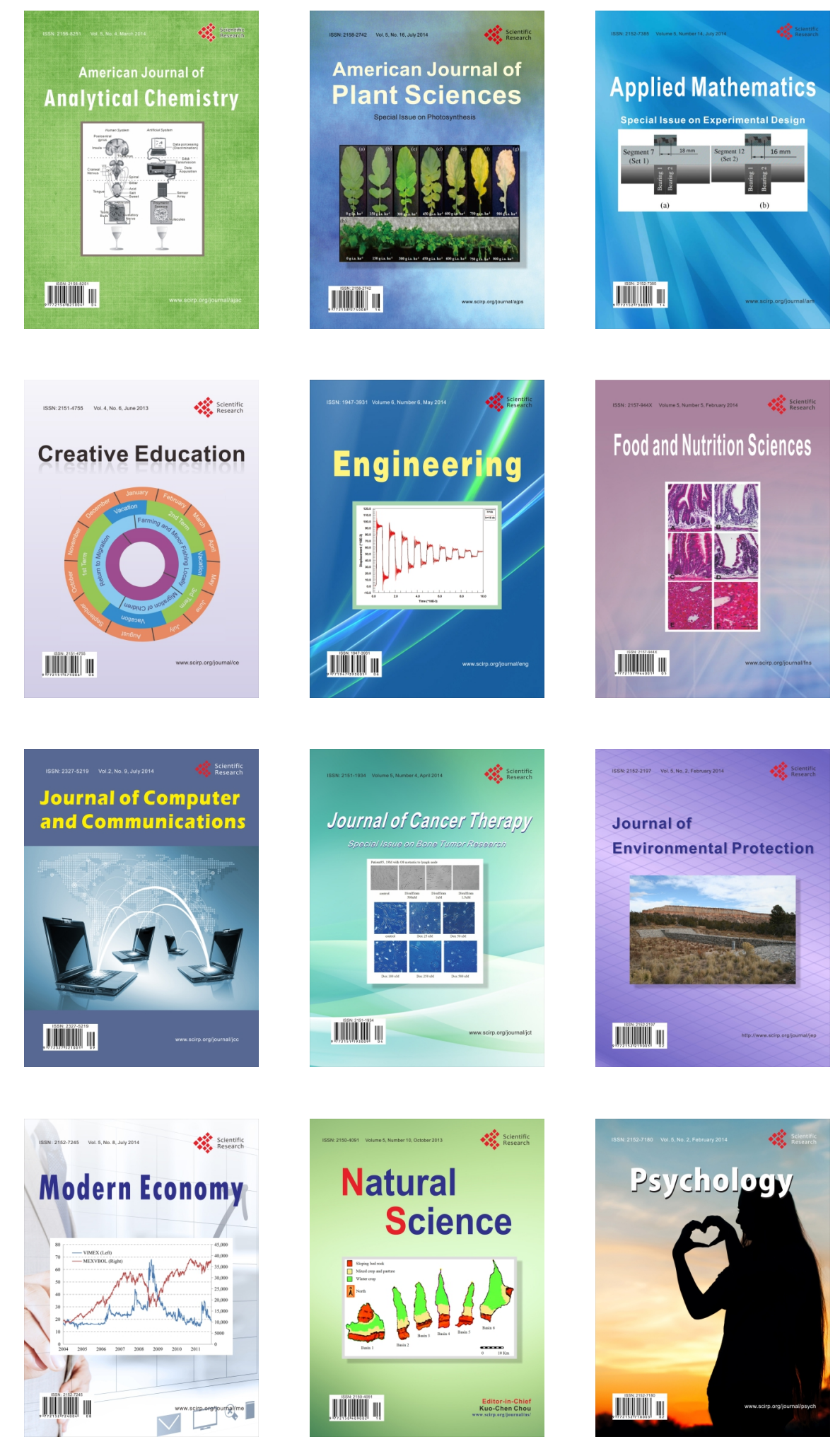